\title{
DEPRESSÃO, ANSIEDADE E QUALIDADE DE VIDA EM IDOSOS DE UMA UNIVERSIDADE ABERTA À TERCEIRA IDADE
}

\section{DEPRESSION, ANXIETY AND QUALITY OF LIFE IN ELDERLY PARTICIPANTS OF AN OPEN UNIVERSITY FOR SENIOR CITIZENS}

\author{
Bruno Felipe Ferreira Lopes ${ }^{1} *$ Thaisy Rodrigues de Oliveira $^{2} *$ Kellen Karoline Almeida dos Santos $^{4}$ \\ Lira $^{3} *$ Gilberto Lima dos Santos ${ }^{4}$ * Glauber Sá Brandão ${ }^{5 \#}$
}

\begin{abstract}
RESUMO
Objetivo: O objetivo deste estudo foi testar a associação entre ansiedade, depressão e a qualidade de vida de idosos participantes de uma Universidade Aberta à Terceira Idade (UATI). Métodos: Trata-se de um estudo descritivo, transversal, com abordagem quantitativa, realizado com idosos $(\geq 60$ anos) sem declínio cognitivo, participantes da UATI em Senhor do Bonfim, BA. Os idosos foram avaliados por meio de três questionários: Escala de Depressão Geriátrica, Inventário de Ansiedade Geriátrica e instrumento de avaliação da qualidade de vida da Organização Mundial da Saúde (WHOQOL-Old). Os dados foram submetidos à análise descritiva e a análise de correlação de Spearman foi utilizada para verificar a associação entre as variáveis. Resultados: dentre os 28 idosos participante, observou-se prevalência de 14,3\% de depressão e $17,9 \%$ de ansiedade. Em relação aos domínios de qualidade de vida, a Participação Social $(77,9 \pm 11,3)$ e Funcionamento do Sensório $(76,3 \pm 17,1)$ apresentaram as maiores médias. A qualidade de vida se correlacionou negativa e moderadamente com a depressão $(-0,439)$ e a ansiedade $(-0,436)$, enquanto que ambos os transtornos apresentaram correlação positiva e moderada $(0,671)$ entre si. Conclusões: Os idosos que obtiveram maior grau de ansiedade e depressão, apresentaram pior qualidade de vida, demonstrando a existência de associação significativa entre estas variáveis. Estes achados direcionam a necessidade de estudos que investiguem o surgimento da ansiedade e depressão em idosos, dissociando estas doenças de processos naturais do envelhecimento.
\end{abstract}

Palavras-chave: Enfermagem geriátrica; Envelhecimento; Qualidade de vida; Depressão; Ansiedade.

\begin{abstract}
Objective: The aim of this study was to test the association between these diseases and the quality of life of elderly participants at an Open University Program for Senior Citizens (UATI). Methods: This is a descriptive, cross-sectional study, with a quantitative approach, carried out with 28 elderly participants of an Open University Program for Senior Citizens, in Senhor do Bonfim, BA. The elderly were assessed using three questionnaires: Geriatric Depression Scale (GDS), Geriatric Anxiety Inventory (GAI) and an instrument for assessing the quality of life of the World Health Organization (WHOQOL-Old). The data were submitted to descriptive analysis and Spearman's correlation analysis was used to verify the association between variables. Results: There was a prevalence of $14.3 \%$ of depression and $17.9 \%$ of anxiety. Social Participation $(77.9 \pm 11.3)$ and Sensory Functioning $(76.3 \pm 17.1)$ presented the highest averages in relation to the quality of life domains. Quality of life was negatively correlated with depression (-0.439) and anxiety (-0.436), while both disorders showed a positive correlation (0.671) with each other. Conclusion: There is a significant relationship between anxiety, depression and quality of life among the elderly surveyed. These findings direct the need for studies that investigate the emergence of anxiety and depression in the elderly, dissociating these diseases from natural aging processes.
\end{abstract}

Keywords: Geriatric Nursing; Aging; Quality of Life; Depression; Anxiety.

\footnotetext{
${ }^{1}$ Universidade do Estado da Bahia, Senhor do Bonfim-BA, Brasil. ORCID: https://orcid.org/0000-0002-5049-7356

${ }^{2}$ Universidade do Estado da Bahia, Senhor do Bonfim-BA, Brasil. ORCID: https://orcid.org/0000-0003-3313-1624

${ }^{3}$ Universidade do Estado da Bahia, Senhor do Bonfim-BA, Brasil. ORCID: https://orcid.org/0000-0003-3121-838X

${ }^{4}$ Universidade do Estado da Bahia, Senhor do Bonfim-BA, Brasil. ORCID: https://orcid.org/0000-0002-6582-724X

${ }^{5}$ Universidade do Estado da Bahia, Senhor do Bonfim-BA, Brasil. ORCID: https://orcid.org/0000-0003-4462-0861

https://doi.org/10.31011/reaid-2021-v.95-n.35-art.1172 Rev Enferm Atual In Derme v. 95, n. 35, 2021 e-021116
} 


\section{INTRODUÇÃO}

O envelhecimento é um processo biológico dinâmico, progressivo e irreversível cujas alterações são caracterizadas por mudanças estruturais e funcionais no corpo humano ${ }^{(1)}$. O processo de envelhecimento da população está expresso no aumento da expectativa de vida, que é decorrente de avanços tecnológicos na área da saúde, alterações na estrutura familiar, nos padrões de trabalho e nas migrações, bem como nas percepções dos indivíduos acerca de aspectos biológicos, culturais, psicológicos e sociais ${ }^{(2)}$.

Segundo a Organização Mundial da Saúde (OMS), o número de pessoas com 60 anos ou mais terá aumentado em aproximadamente $\quad 70 \%$ até 2025, especialmente nos países em desenvolvimento (3). No Brasil, são estimadas cerca de 32,9 milhões de pessoas com 65 anos ou mais, com projeção de curva de crescimento para 58,2 milhões até 2060 , o equivalente a $25,5 \%$ da população ${ }^{(2)}$.

$\mathrm{Na}$ atualidade é verificada a elevação na incidência de distúrbios psicológicos em idosos. A OMS estima que aproximadamente um em cada 10 idosos pode sofrer de depressão ${ }^{(4)}$.

Em pessoas idosas, a depressão é o transtorno psiquiátrico mais frequente, e está associada ao maior risco de morbidade, mortalidade e falta de controle sobre o próprio estado emocional ${ }^{(5)}$. Ainda assim, é uma doença frequentemente subdiagnosticada e subtratada por ter semelhanças com complicações físicas e mentais, consideradas naturais ao envelhecimento ${ }^{(6)}$.

A ansiedade é associada às limitações presentes na velhice, ocasionando percepções negativas da realidade e questionamentos acerca das próprias habilidades intelectuais. Altos níveis de ansiedade em idosos ocasionam vivências, que interferem na atenção seletiva e na codificação de informações na memória, bloqueando assim a compreensão e o raciocínio ${ }^{(7)}$.

A qualidade de vida é o conjunto multidimensional e multifatorial de satisfações subjetivas que os sujeitos obtêm no seu dia a dia, que considera os aspectos físicos, psicológicos e sociais da vida, sendo útil para determinar o impacto global das doenças e dos tratamentos médicos a partir da perspectiva do indivíduo ${ }^{(8)}$. Sendo assim e, considerando que os transtornos de ansiedade e depressão tendem a diminuir a qualidade de vida, compreender a relação entre estas condições é especialmente importante em idosos, uma vez que estes distúrbios podem afetar as relações interpessoais, o suporte físico, social e o desempenho cognitivo, o que impacta negativamente sobre a qualidade de vida ${ }^{(9)}$. No entanto, há poucas investigações a 
respeito da relação entre estes transtornos psiquiátricos e a qualidade de vida na população idosa ${ }^{(10)}$.

O objetivo deste estudo foi testar a associação entre ansiedade, depressão e a qualidade de vida de idosos.

\section{MÉTODOS}

\section{Participantes, local e tipo de estudo}

Trata-se de um estudo descritivo, transversal, com abordagem quantitativa. $\mathrm{O}$ procedimento de amostragem utilizado foi $\mathrm{o}$ não probabilístico por conveniência, sendo a amostra constituída por idosos cadastrados no programa Universidade Aberta da Terceira Idade - UATI da Universidade do Estado da Bahia - UNEB, em Senhor do Bonfim, BA. Esta pesquisa foi realizada a partir de dados provenientes de um projeto maior que tem como objetivo verificar o efeito de um programa de abordagem multidisciplinar na qualidade de vida de idosos da UATI, o qual foi desenvolvido por pesquisadores vinculados ao grupo de estudos e pesquisa em qualidade de vida e envelhecimento saudável ( QualES) da UNEB-BA, Brasil. O desenho e a condução do presente estudo seguiram as recomendações do Reporting of Observational Studies in Epidemiology (STROBE).
Como critérios de inclusão, definiu-se que os participantes foram idosos (60 anos ou mais), de ambos os sexos, matriculados na UATI. Como critério de exclusão, considerou-se a presença de declínio cognitivo de acordo com o Mini Exame do Estado Mental (MEEM) ${ }^{(11)}$.

\section{Instrumentos de avaliação dos participantes}

Foi aplicada uma ficha de identificação para coleta dos dados sociodemográficos: sexo, idade, estado civil, escolaridade, arranjo familiar e renda.

\section{Escala de Depressão Geriátrica}

A depressão foi avaliada por meio da Escala de Depressão Geriátrica (EDG-15), uma versão simplificada da escala original, utilizada amplamente para o rastreamento de sintomas depressivos na população idosa, tanto na identificação do quadro quanto no monitoramento de mudanças ao longo do tempo. Pode ser auto aplicada ou aplicada por um entrevistador ${ }^{(12)}$. A escala possui uma variação de zero (ausência de sintomas depressivos) a quinze pontos (pontuação máxima de sintomas depressivos), para qual os autores ${ }^{(13)}$ definiram o ponto de corte igual ou maior que cinco para determinar a presença de sintomas depressivos nos idosos. 


\section{Inventário de Ansiedade Geriátrica}

O Inventário de Ansiedade Geriátrica (GAI) é um instrumento para avaliação de ansiedade em idosos nos contextos geriátricos e comunitários, proposto pelo estudo ${ }^{(14)}$. Este questionário é composto por 20 questões, em que o respondente declara concordar ou discordar das afirmações apresentadas. A somatória das respostas concordo $>10$ caracteriza o indivíduo com sintomatologia de Ansiedade. Pode ser administrado de forma auto aplicada, ou aplicada pelo entrevistador.

\section{WHOQOL-Old}

Para avaliação da qualidade de vida dos idosos, foi utilizado o instrumento do Grupo de Qualidade de Vida da Organização Mundial de Saúde (WHOQOL-Old) ${ }^{(15)}$. Este instrumento é constituído de 24 perguntas, atribuídas a seis facetas: (I) Funcionamento do Sensório, (II) Autonomia, (III) Atividades Passadas, Presentes e Futuras, (IV) Participação Social, (V) Morte e Morrer e (VI) Intimidade. Cada uma das facetas possui quatro perguntas, cujas respostas seguem uma escala de Likert (1-5). Para todas as facetas, o escore dos valores possíveis pode oscilar de 0 a 100, onde maiores valores representam uma maior qualidade de vida.

\section{Coleta e análise de dados}

A coleta de dados foi realizada entre novembro e dezembro de 2019. Os questionários foram aplicados sob forma de entrevista, de forma clara e objetiva, sem que ocorresse interferência do entrevistador na resposta. Em caso de dúvidas na compreensão pelo idoso, o entrevistador repetiu a questão até que ocorresse a compreensão por parte do entrevistado.

Este estudo foi aprovado pelo Comitê de Ética em pesquisa envolvendo seres humanos da Universidade do Estado da Bahia, com CAAE: 25875819.8.0000.0057. Todos os participantes assinaram um Termo de Consentimento Livre e Esclarecido (TCLE). Os princípios éticos gerais foram acatados considerando-se que a pesquisa em qualquer área do conhecimento, envolvendo seres humanos, deve ser coerente com os princípios da ciência. Este estudo assegurou o cumprimento dos princípios éticos da resolução 466/12 do Conselho Nacional de Saúde.

Os dados foram testados quanto à normalidade, em seguida foram submetidos à análise descritiva por meio de frequências absolutas e percentuais para variáveis categóricas e medidas de tendência central e dispersão para variáveis numéricas. Para realizar a análise de correlação, em decorrência da identificação da não normalidade dos dados, optou-se em utilizar o teste não paramétrico $(\rho)$ de Spearman para verificar a associação entre as variáveis 
depressão, ansiedade e qualidade de vida. Para critérios de decisão, foi adotado o nível de significância $\alpha \leq 0,05$ e as análises foram realizadas no software Statistical Package of the Social Sciences (SPSS 21.0, IBM, EUA).

\section{RESULTADOS}

A amostra do presente estudo foi composta por 28 idosos, predominantemente do sexo feminino $(\mathrm{n}=27 ; 96,4 \%)$, com idade variando entre 60 e 86 anos (média de 68,6 $6,2)$, sendo a maioria viúvas $(\mathrm{n}=14 ; 50,0 \%)$, com ensino fundamental $(\mathrm{n}=16 ; 57,1 \%)$ e com renda mensal de até um salário mínimo ( $\mathrm{n}=15 ; 53,6 \%)$, conforme apresentado na Tabela 1. Todas participantes do programa Universidade Aberta à Terceira Idade.

Tabela 1 - Variáveis sociodemográficas dos idosos participantes $(n=28)$. Senhor do Bonfim, BA, 2019.

\begin{tabular}{|c|c|c|}
\hline Variável sociodemográfica & $\mathrm{n}$ & $\%$ \\
\hline \multicolumn{3}{|l|}{ Sexo } \\
\hline Feminino & 27 & 96,4 \\
\hline \multicolumn{3}{|l|}{ Idade } \\
\hline 60-69 anos & 18 & 64,3 \\
\hline $70+$ anos & 10 & 35,7 \\
\hline \multicolumn{3}{|l|}{ Estado civil } \\
\hline Solteiro (a) & 4 & 14,3 \\
\hline Casado (a) & 10 & 35,7 \\
\hline Viúvo (a) & 14 & 50,0 \\
\hline \multicolumn{3}{|l|}{ Escolaridade } \\
\hline Ensino fundamental & 16 & 57,1 \\
\hline Ensino médio & 9 & 32,1 \\
\hline Ensino superior & 3 & 10,7 \\
\hline \multicolumn{3}{|l|}{ Arranjo domiciliar } \\
\hline Moram só & 9 & 32,1 \\
\hline Moram com cônjuge ou parentes próximos & 19 & 67,9 \\
\hline \multicolumn{3}{|l|}{ Renda } \\
\hline Até 1 salário mínimo & 15 & 53,6 \\
\hline 2-3 salários mínimos & 10 & 35,7 \\
\hline 4 ou mais salários mínimos & 3 & 10,7 \\
\hline
\end{tabular}


Fonte: Os autores.

A tabela 2 apresenta a estatística descritiva para os seis domínios da qualidade de vida avaliada nos idosos pelo questionário WHOQOL-Old. Cada domínio do questionário é composto por quatro perguntas.
$(65,6 \pm 19,1)$ e Autonomia $(67,0 \pm 11,0)$ apresentaram as menores médias, enquanto Participação Social $\quad(77,9 \pm 11,3)$ e Funcionamento do Sensório $(76,3 \pm 17,1)$ as maiores médias.

Dentre os seis domínios, Morte e Morrer

Tabela 2 - Estatística descritiva das seis facetas do questionário WHOQOL-Old avaliada em idosos $(\mathrm{n}=28)$. Senhor do Bonfim, BA, 2019.

\begin{tabular}{lccc}
\hline Domínio & Média \pm DP & Mínimo & Máximo \\
\hline Funcionamento do Sensório & $76,3 \pm 17,1$ & 43,8 & 100,0 \\
Autonomia & $67,0 \pm 11,0$ & 50,0 & 81,3 \\
Atividades Passadas, Presentes e Futuras & $74,3 \pm 17,1$ & 43,8 & 100,0 \\
Participação Social & $77,9 \pm 11,3$ & 56,3 & 100,0 \\
Morte e Morrer & $65,6 \pm 19,1$ & 12,5 & 87,5 \\
Intimidade & $68,5 \pm 21,9$ & 25,0 & 100,0 \\
\hline Média geral & $71,6 \pm 10,8$ & & \\
\hline
\end{tabular}

Fonte: Os autores.

A partir dos pontos de corte sugeridos pelos autores ${ }^{(13)}$ para depressão e pelos autores ${ }^{(14)}$ para ansiedade, observou-se prevalência de
14,3\% de idosos com depressão e 17,9\% com ansiedade, assim como demonstrado na figura 1 .

Figura 1 - Prevalência de depressão e ansiedade dos idosos participantes $(n=28)$. Senhor do Bonfim, BA, 2019.

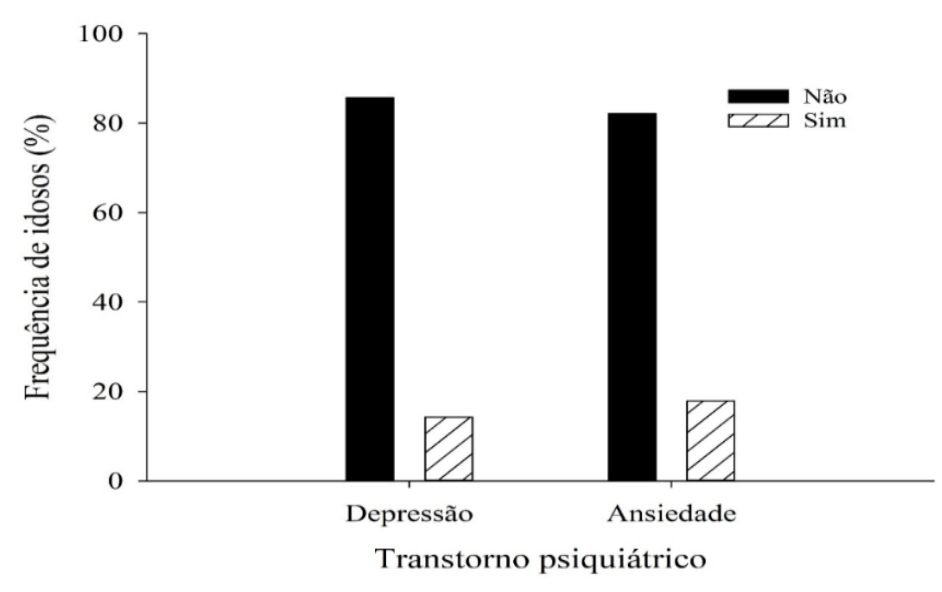

Fonte: Autores ${ }^{(13,14)}$ 
A associação entre ansiedade, moderadamente com a depressão $(\rho=-0,439$; depressão e qualidade de vida foi avaliada $p=0,02$ e a ansiedade $(\rho=-0,436 ; p=0,021)$, pela correlação de Spearman, cujos valores enquanto que ambos os transtornos são apresentados na tabela 3. A qualidade de apresentaram correlação positiva e moderada vida se correlacionou negativa e $(\rho=0,671 ; p<0,001)$ entre si.

Tabela 3 - Correlação de Spearman entre depressão, ansiedade e qualidade de vida dos idosos participantes $(\mathrm{n}=28)$. Senhor do Bonfim, BA, 2019.

\begin{tabular}{lccc}
\hline & & Ansiedade & Qualidade de vida \\
\hline \multirow{2}{*}{ Depressão } & $\rho$ & 0,671 & $-0,439$ \\
& p-valor & $<0,0001$ & 0,0196 \\
\multirow{2}{*}{ Ansiedade } & $\rho$ & & $-0,436$ \\
& p-valor & & 0,0207 \\
\hline
\end{tabular}

Fonte: Os autores

\section{DISCUSSÃO}

A participação de idosos em programas intervencionistas de educação em saúde e autocuidado tem impacto positivo na promoção da saúde mental e estilos de vida saudável, bem como na prevenção de declínios físicos e cognitivos ${ }^{(16)}$. Um exemplo de um programa deste género é a Universidade Abertas à Terceira Idade (UATI) que se apresenta como alternativa de promoção à reinserção psicossocial e ao desenvolvimento de ações educativas de caráter permanente.

Neste estudo, conduzido na UATI de Senhor do Bonfim, BA, a amostra foi constituída, em sua maioria, por mulheres, viúvas, com ensino fundamental e com renda mensal de até um salário mínimo. Uma provável explicação para a predominância feminina está descrita em estudo socioepidemiológico demográfico em uma UATI do estado de Pernambuco, no qual os autores ${ }^{(17)}$ relacionam as diferenças quanto à percepção do envelhecimento e como os indivíduos vivenciam tais mudanças, sendo que mulheres demonstraram maior interesse em cultura, bem estar e saúde e homens interesse em assuntos políticos.

No que se refere ao arranjo familiar, a maioria dos participantes convivia com o cônjuge e/ou parentes próximos, incluindo filhos e netos. Estes dados trazem aspectos positivos relacionados à saúde, pois segundo estudo realizado ${ }^{(18)}$, o suporte familiar tende a reduzir os efeitos negativos do estresse na 
saúde mental, possibilitando assim, uma influência positiva no bem-estar psicológico. Em seus estudos, os autores ${ }^{(19)}$, sugerem que a interação social reduz $\mathrm{o}$ isolamento $\mathrm{e}$ oferece ao idoso a estimulação do desempenho cognitivo, aumentando sua satisfação e melhorando a qualidade de vida.

Neste estudo foi observado que a média geral dos domínios de qualidade de vida do questionário WHOQOL-Old foi alta, destacando que os domínios relacionados à participação social e funcionamento do sensório obtiveram as maiores médias, o que pode ser reflexo da participação em um programa intervencionista de promoção à saúde e bem-estar, com a prática de exercício físico regular e intervenções que estimularam o desempenho mnemônico. Em um estudo realizado $^{(20)}$, foi observado que idosos participantes de um programa regular de exercícios físicos mostraram melhora significativa na mobilidade funcional e na qualidade de vida. Para os autores ${ }^{(21)}$ o exercício físico diminui o declínio funcional, especialmente as atividades aeróbicas e de resistência. O estudo (22) afirma que intervenções com o objetivo de melhorar o desempenho mnemônico, propiciam a manutenção da capacidade funcional, minimizando os riscos de dependência e possível institucionalização da pessoa idosa.
A prevalência das doenças psiquiátricas investigadas neste estudo é menor do que aquelas observadas ${ }^{(23,24)}$, em indivíduos brasileiros da comunidade, com características sociodemográficas similares, que variou entre $29,6 \%$ e $47,4 \%$. A população estudada apresentou baixa prevalência de depressão (14,3\%) em comparação a estudos brasileiros realizados com idosos da Atenção Primária à Saúde, com variações entre 26,1\% a $30,6 \%{ }^{(25-27)}$. Em estudo realizado ${ }^{(28)}$ afirma que idosos com níveis mais elevados de desenvolvimento nas relações sociais são mais ativos e têm melhor qualidade de vida e menos sintomas depressivos do que idosos sedentários e isolados socialmente.

A qualidade de vida é um conceito multifatorial e pode ser influenciada por diferentes circunstâncias, ocasionando distintos níveis de ansiedade, o conceito de ansiedade pode ser entendido como um sentimento desagradável, relacionado a percepções negativas de antecipação de perigo, ocasionando desconforto e tensão. Desse modo, é importante compreender as influências da ansiedade sobre a saúde do idoso para que não ocorra confusão entre a detecção e o diagnóstico de transtornos de ansiedade com sintomas físicos comuns na terceira idade ${ }^{(28)}$.

Destaca-se a correlação negativa entre ansiedade e qualidade de vida nos idosos, em 
concordância com resultados encontrados na literatura em estudos realizados pelos autores ${ }^{(9,29)}$, os quais observaram que há uma associação entre transtornos de ansiedade e a piora da qualidade de vida, além de maior uso de serviços de saúde. Indivíduos com altos níveis de ansiedade obtiveram piores escores de qualidade de vida em campos como o da vitalidade, aspectos sociais e retraimento social $^{(30)}$.

Foi possível verificar a associação negativa, moderada e estatisticamente significativa entre qualidade de vida $\mathrm{e}$ depressão em alinhamento com os resultados de um estudo brasileiro realizado pelos autores (31), que observaram piora na qualidade de vida em domínios como autonomia, atividades, intimidade, e participação social em idosos depressivos, em comparação com idosos sem sintomas de depressão. Os autores (30), verificaram que indivíduos em estado depressivo tem pouca interação social e utilizam mais os serviços de saúde.

Constatou-se uma correlação positiva, moderada e estatisticamente significativa entre ansiedade e depressão. Os autores (32) destacam que indivíduos acometidos no mesmo período por ansiedade e depressão, sofreram aumento na ocorrência de distúrbios físicos, incluindo problemas de visão, tosse persistente, hipertensão e problemas gastrointestinais, além de doenças cardíacas e asma, em comparação àqueles que sofriam apenas com ansiedade e apenas com depressão. De acordo com os autores (7), indivíduos com sintomas depressivos avaliam sua saúde como ruim e estão menos satisfeitos com seu estado de saúde do que pessoas sem depressão. Além disso, os autores reportam que é comum portadores de ansiedade também apresentarem depressão em alguma intensidade.

São muitos os estudos que indicam a carência de investigação destas doenças psiquiátricas por parte dos profissionais de saúde no contexto clínico, embora sejam comuns como queixas clínicas apresentadas pelos pacientes $^{(7)}$. É importante a avaliação clínica e laboratorial da ansiedade e da depressão, visto que é preciso investigar doenças que podem estar associadas a estes transtornos, de modo que, se não forem compreendidas e tratadas de modo adequado, podem ocasionar repercussões negativas na saúde e na qualidade de vida de pessoas idosas, podendo levar a um aumento da morbidade e da mortalidade, como também a elevação de custos para o sistema de saúde (33).

Apesar de pequena, a amostra é representativa e vislumbra o desfecho situacional local, tendo em vista que idosos 
participantes de programas intervencionistas tendem a não formar grupos grandes.

Este estudo apresenta algumas limitações como a utilização de um delineamento transversal, onde a associações entre as variáveis deve ser interpretada com cautela, pois, em estudos de associação não é possível realizar inferência causal. Outra importante limitação está relacionada ao fato de que, embora tenham sido utilizados questionários validados, mas a utilização de medidas autorreferidas, aumenta a possibilidade do erro de aferição, do viés de recordação e o efeito do desejo social.

\section{CONCLUSÕES}

Foi observado que ansiedade e depressão se correlacionam positivamente entre si e negativamente com a qualidade de vida. Há relação estatisticamente significativa e moderada entre os três aspectos supracitados. Logo, torna-se compreensível a intrínseca relação entre os transtornos psicológicos e seus efeitos na saúde do idoso de forma integral, uma vez que este começa a buscar mais os serviços de saúde. O diagnóstico e tratamento adequados da depressão e ansiedade podem melhorar a qualidade de vida dos idosos. Em se tratando dos domínios da qualidade de vida, a participação social e o funcionamento do sensório apresentaram as maiores médias para os idosos participantes do estudo, evidenciando a efetividade de programas intervencionistas, como a UATI, em prover melhora da qualidade de vida.

\section{REFERÊNCIAS}

1. Pinheiro LCN, Aguiar MIB, Nunes AAA, Araujo KC, Pessoa CN. Conhecimento e hábitos da fotoproteção na geriatria em uma instituição filantrópica. Mostra Cient Farm. 2019;5(1).

2. Instituto Brasileiro de Geografia e Estatística. Idosos indicam caminhos para uma melhor idade [Internet]. Brasília: IBGE; 2020 [cited 2021 Apr 10]. Available from: http://www.ibge.gov.br

3. Organização Mundial da Saúde. Envelhecimento e saúde [Internet]. Brasília: IBGE; 2018 [cited 2021 Apr 11]. Available from: https://www.paho.org/pt/brasil

4. World Health Organization. Depression: evidence-based recommendations for management of depression in nonspecialized health settings [Internet]. 2015 [cited 2021 Apr 11]. Available from: www.who.int/mental_health/mhgap/evidence/ depression/en

5. Sarokhani D, Parvareh M, Dehkordi AH, Sayehmiri K, Moghimbeigi A. Prevalence of depression among iranian elderly: systematic review and meta-analysis. Iran J Psychiatry. 2018;13(1):55.

6. Zou C, Chen S, Shen J, Zheng X, Wang L, Guan L, Liu Q, Yang Y. Prevalence and associated factors of depressive symptoms among elderly inpatients of a Chinese tertiary hospital. Clin Interv Aging. 2018;13(13):1755-62.

7. Oliveira KL, Santos AAA, Cruvinel M, Néri AL. Relação entre ansiedade, depressão e desesperança entre grupos de idosos. Psicol Estud. 2006;11(2):351-9, 2006.

8. Oliveira, LSSCB, Souza EC, Rodrigues RAS, Fett CA, Piva AB. The 
effects of physical activity on anxiety, depression, and quality of life in elderly people living in the community. Trends Psychiatry Psychother. 2019;41(1): 36-42.

9. Oliveira DV, Antunes MD, Oliveira J. Ansiedade e sua relação com a qualidade de vida em idosos: revisão narrativa. Cinergis. 2017;18(4):316-22.

10. Kang HJ, Bae KY, Kim SW, Shin HY, Shin IS, Yoon JS, Kim JM. Impact of Anxiety and Depression on Physical Health Condition and Disability in an Elderly Korean Population. Psychiatry Investig. 2017;14(3):240-8.

11. Folstein MF, Folstein SE, McHugh PR. "Mini-mental state". A practical method for grading the cognitive state of patients for the clinician. J Psychiatr Res. 1975;12(3):18998 .

12. Sheikh JI, Yesavage JA. Geriatric Depression Scale (GDS): recent evidence and development of a shorter version. Clin Gerontol. 1986;5(1-2):165-73.

13. Almeida OP, Almeida SA. Confiabilidade da versão brasileira da Escala de Depressão em Geriatria (GDS) versão reduzida. Arq Neuropsiquiatr. 1999;57(2):421-6.

14. Pachana NA, Byrne GJ, Siddle H, Koloski N, Harley E, Arnold E. Development and validation of the Geriatric Anxiety Inventory. Int Psychogeriatr. 2007;19(1):10314.

15. Power M, Quinn K, Schmidt S; WHOQOL-OLD Group. Development of the WHOQOL-old module. Qual Life Res. 2005;14(10):2197-214.

16. Loureiro APF. Desafios do envelhecimento populacional: por uma educação permanente participada. Laplage Rev. 2019;5(2):42-49.

17. Barreto KML, Carvalho EMFD, Falcão IV, Lessa FJD, Leite VMM. Perfil sócio-epidemiológico demográfico das mulheres idosas da Universidade Aberta à
Terceira Idade no estado de Pernambuco. Rev Bras Saúde Matern Infant. 2003;3(3):339-54.

18. Assis LPP, Amaral MLN. Envelhecimento e suporte social. In: Costa GA, organizator. Atividade física, envelhecimento e a manutenção da saúde. Uberlândia: EDUFU; 2010. p. 207-17.

19. Yassuda MS, Silva HS. Participação em programas para a terceira idade: impacto sobre a cognição, humor e satisfação com a vida. Estud Psicol. 2010;27(2):207-14.

20. Mikkelsen K, Stojanovska L, Polenakovic M, Bosevski M, Apostolopoulos V. Exercise and mental health. Maturitas. 2017;106:48-56.

21. Miranda GMD, Mendes ACG, Silva ALA. $O$ envelhecimento populacional brasileiro: desafios e consequências sociais atuais e futuras. Rev Bras Geriatr Gerontol. 2016;19(3):507-19.

22. Pinto LLT, Rocha SV, Viana HPS, Rodrigues WKM, Vasconcelos LRC. Nível de atividade física habitual e transtornos mentais comuns entre idosos residentes em áreas rurais. Rev Bras Geriatr Gerontol. 2014;17(4):819-28.

23. Rocha SV, Almeida MMG, Araújo TM, Virtuoso Júnior JS. Atividade física no lazer e transtornos mentais comuns entre idosos residentes em um município do nordeste do Brasil. J Bras Psiquiatr. 2011;60(2):80-5.

24. Lino VT, Portela MC, Camachio LA, Atie S, Lima MJ, Rodrigues NC, Barros MBL, Andrade MKN. Screening for depression in low-income elderly patients at the primary care level: use of the patient health questionnaire-2. PLoS One. 2014;9(12):e113778.

25. Lopes NE, Librelotto RL, Souza GS, Irenio G, Alfredo CN. Screening for depressive symptoms in older adults in the Family Health Strategy, Porto Alegre, Brazil. Rev Saúde Públ. 2014;48(3):368-77. 
26. Souza KA, Freitas FFQ, Castro AP, Oliveira CDB, Almeida AAB, Souza KA. Prevalence of depression symptoms in elderly people assisted by the Family Health Strategy. Rev Min Enferm. 2017;21(1):e1018.

27. Oliveira LSSCB, Souza EC, Rodrigues RAS, Fett CA, Piva AB. The effects of physical activity on anxiety, depression, and quality of life in elderly people living in the community. Trends Psychiatry Psychother. 2019;41(1):36-42.

28. Fazzi L, Dobrianskyj FM, Reggi JR, Otani VH, Otani TZ, Uchida RR. The influence of anxiety on quality of life among patients with an indication for cataract surgery. Sao Paulo Med J. 2015;133(2):160-1.

29. Gonçalves AMC, Teixeira MTB, Gama JRA, Lopes CS, Silva GA, Gamarra CJ, Duque KCD, Machado MLSM. Prevalência de depressão e fatores associados em mulheres atendidas pela Estratégia de Saúde da Família. J Bras Psiquiatr. 2018;67(2):101-109.

30. Scherrer Júnior G, Okuno MFP, Oliveira LM, Barbosa DA, Alonso AC, Fram DS, Belasco AGS. Quality of life of institutionalized aged with and without symptoms of depression. Rev Bras Enferm. 2019;72(2):127-133.

31. Kang HJ, Bae KY, Kim SW, Shin HY, Shin IS, Yoon JS, Kim JM. Impact of anxiety and depression on physical health condition and disability in an elderly Korean population. Psychiatry Investig. 2017;14(3):240-248.

32. Andrews JA, Astell AJ, Brown LJE, Harrison RF, Hawley MS. Technology for Early Detection of Depression and Anxiety in Older People. Stud Health Technol Inform. 2017;242:374-380.

Submissão: 2021-07-12

Aprovado: 2021-08-02

\#Autor correspondente: Glauber Sá Brandão. Rodovia Lomanto Júnior, BR 407, Km 127, Senhor do Bonfim, BA, CEP 48970000, Brasil. Telefone: +55 74 98121-3811. Email:gbrandao@uneb.br 